\title{
ON A THEOREM OF BRAUER
}

T. A. BURTON

1. In [1] F. Brauer considers two systems of differential equations:

$$
x^{\prime}=A x+p(t)
$$

and

$$
y^{\prime}=A y+f(t, y)+p(t)
$$

where $A$ is an $n \times n$ matrix, $p$ is an $n$-dimensional column vector function continuous for $0 \leqq t<\infty$, and $f$ is an $n$-dimensional column vector function continuous for $0 \leqq t<\infty,|y|<\infty$. The solutions of (1) are compared with those of (2). He requires that there exist a continuous function $\lambda$ such that

(3) $|f(t, y)|<\lambda(t)|y|, 0 \leqq t<\infty,|y|<\infty$, with $\int_{0}^{\infty} \lambda(t) d t<\infty$.

By interpreting (3) in a certain manner we are able to obtain a stability criterion which is generally stronger than Brauer obtained.

2. Consider the system

$$
z^{\prime}=f(t, z)
$$

and assume a Liapunov function of the form $V(z)=|z|^{2}$, where $|z|$ is taken to be the usual Euclidean length. Then

$$
\frac{d V}{d t}=V^{\prime}=2\left(z_{1} f_{1}+\cdots+z_{n} f_{n}\right)
$$

and $\left|V^{\prime}\right| \leqq 2 \lambda(t)|z|^{2}=2 \lambda(t) V=2 \lambda(t)|V|$. Integrating $\left|V^{\prime}\right| \leqq 2 \lambda(t)|V|$ from 0 to $t$ we obtain $|V| \leqq \exp 2 \int_{0}^{t} \lambda(s) d s<\infty$ by (3). But $V(t)$ being bounded implies that every solution of $z^{\prime}=f(t, z)$ is bounded.

This leads us to the following considerations. In (1) and (2) let $p(t) \equiv 0$ and let the characteristic roots of $A$ have negative real parts. Then there exists a positive definite matrix $C[2$, p. 26] such that $V(x)=x^{T} C x$ is a Liapunov function for (1) and $V^{\prime}=x^{T}\left[C A+A^{T} C\right] x$ has the property that $C A+A^{T} C=-D$ where $D$ is positive definite. Now let us use $V(y)=y^{T} C y$ for a Liapunov function for (2). We have

$$
\begin{aligned}
V^{\prime}(y) & =y^{T} C y+y^{T} C y^{\prime}=(A y+f)^{T} C y+y^{T} C(A y+f) \\
& =y^{T} A^{T} C y+f^{T} C y+y^{T} C A y+y^{T} C F=-y^{T} D y+f^{T} C y+y^{T} C f .
\end{aligned}
$$

Received by the editors April 19, 1965. 
A positive number $k$ can be found such that $-y^{T} D y \leqq-k|y|^{2}$.

THEOREM. Suppose there exist a continuous function $\lambda$ on $0 \leqq t<\infty$ and a number $M$ such that $y^{T} C f$ and $f^{T} C y$ are each less than $\lambda(t)|y|^{2} /(2+\epsilon)$ for $t>M$ and $\epsilon>0$, but $\epsilon$ as small as we please. If $\lambda(t)<k$ for $t>M$, then every solution of (2) tends to zero as $t \rightarrow \infty$. If $M=0$, then the null solution of (2) is globally asymptotically stable.

The proof is immediate if $M=0$ since $V^{\prime}$ is negative definite. If $M>0$, then all we must show is that no solution has finite escape time and this is easily done using arguments similar to those at the start of this section.

Since we require that $\lambda(t)<k$ for $t$ sufficiently large while Brauer requires that $\int_{0}^{\infty} \lambda(t) d t<\infty$, the conditions are not strictly comparable, but in general the former is less restrictive. Also, the requirement that $p(t) \equiv 0$ is slight due to our conditions on $f$ and $\lambda$.

\section{BIBLIOGRAPHY}

1. F. Brauer, Nonlinear differential equations with forcing terms, Proc. Amer. Math. Soc. 15 (1964), 758-765.

2. W. Hahn, Theory and application of Liapunov's direct method. Prentice-Hall Englewood Cliffs, N. J., 1963.

University of Alberta, Edmonton 